\section{Evaluating digital diabetic retinopathy screening in people
aged 90 years and over screening in people
aged 90 years and over}

${ }^{1}$ Departments of Diabetes and Ophthalmology, Heartlands Hospital, Birmingham, UK

${ }^{2}$ School of Health and Life Sciences, Aston University, Birmingham, UK

${ }^{3}$ Birmingham and Midland Eye Centre, City Hospital, Birmingham, UK

Correspondence:

A Tye, Diabetes \& Endocrinology Centre, Birmingham Heartlands Hospital, Bordesley Green East, Birmingham B9 5SS, UK

Tel: +44 (0)12 1424 2039;

Fax: +44 (0)1214242982.

E-mail: abigail.tye@

heartofengland.nhs.uk

Received: 15 January 2015 Accepted in revised form:

6 June 2015

Published online:

17 July 2015

Previously presented at Diabetes UK and The Royal College of Ophthalmologists conferences.

\begin{abstract}
Purpose To evaluate the effectiveness of digital diabetic retinopathy screening in patients aged 90 years and over. Methods This is a retrospective analysis of 200 randomly selected patients eligible for diabetic retinopathy screening aged 90 years and over within the Birmingham, Solihull, and Black Country Screening Programme. Results One hundred and seventy-nine $\mathbf{( 9 0 \% )}$ patients attended screening at least once. Outcomes: 133 (74\%) annual screening after their first screen, of whom $59 \%$ had no detectable diabetic retinopathy; $38(21 \%)$ were referred for ophthalmology clinical assessment- 36 for nondiabetic retinopathy reasons and two for diabetic maculopathy. Cataract accounted for $50 \%$ of all referrals for ophthalmology clinical assessment. Of the 133 patients placed on annual screening, $93(70 \%)$ were screened at least once more. In terms of level of diabetic retinopathy, assessability or other ocular pathologies, 8 improved, 51 remained stable, and 31 deteriorated. Of the latter, 19 patients were referred for ophthalmology clinical assessment; none of these for diabetic retinopathy. Conclusions Screening provides opportunistic identification of important nondiabetic retinopathy eye conditions. However, in view of the low identification rate of sight-threatening diabetic retinopathy in patients aged 90 years and over, and the current mission statement of the NHS Diabetic Eye Screening Programme, systematic annual diabetic retinopathy screening may not be justified in this age group of patients, but rather be performed in optometric practice.

Eye (2015) 29, 1442-1445; doi:10.1038/eye.2015.130; published online 17 July 2015

\section{Introduction}

Diabetic retinopathy (DR) is a common cause of blindness in the United Kingdom and all people
\end{abstract}

A Tye ${ }^{1}$, H Wharton ${ }^{1}$, A Wright ${ }^{1}$, Y Yang ${ }^{2,3}$, J Gibson ${ }^{1,2}$, A Syed ${ }^{1}$, A Mills ${ }^{1}$ and P Dodson ${ }^{1,2}$ with diabetes are at risk of developing the disease. In 2012, the known UK population with diagnosed diabetes mellitus (DM) was reported to be $>3.8$ million people. ${ }^{1}$ Future projections from epidemiologic data show that the prevalence of DM in the elderly population is an increasing significant public health burden. ${ }^{2,3}$

In terms of chronic diseases, DM is one of the commonest for elderly people ${ }^{4}$ and has serious complications such as coronary heart disease, nephropathy, neuropathy, and cognitive dysfunction. Subsequently, they often require different management to younger people with DM.

Elderly persons are also very prone to developing visual impairment, ${ }^{5}$ with important causes being age-related macular degeneration (AMD), glaucoma, and cataract. ${ }^{5}$ Proliferative DR is strongly associated with vision loss and duration of diabetes is an important factor. ${ }^{6-8}$ However, previous research suggests that DR is not clearly related to advanced age. ${ }^{9}$ Although the prevalence of DM is fairly high in elderly persons, the incidence of DR remains relatively low. ${ }^{10,11}$ Cahill et al ${ }^{10}$ reported a $14 \%$ prevalence of DR in their cohort of patients diagnosed with DM after the age of 70 years. More recently, Xin and Zhaoyan ${ }^{11}$ found a DR prevalence of $15.38-16.20 \%$ and an incidence of 8.38/1000 person-years in their study of 2194 elderly subjects aged between 60 and 97 years (mean age of 72.5 years). Furthermore, progression from background to proliferative retinopathy has been found in some studies to be less common in the elderly, ${ }^{7}$ including neovascularisation of the disc. ${ }^{12}$

The NHS Diabetic Eye Screening Programme currently advises that all people with diabetes aged 12 years and over should be screened annually for DR. UK screening programmes for other conditions have upper age limits but this has not been considered previously for Diabetic Eye Screening. Therefore, the primary aim of this 
study was to evaluate the value of digital DR screening in patients aged 90 years and over in order to establish whether it is worthwhile to screen at age 90 years and above.

\section{Materials and methods}

A search on the Digital Healthcare database of the Birmingham, Solihull and Black Country Screening Programme was performed in April 2011. All patients who were 90 years or older at the time of the database search were identified. Two-hundred patients were randomly selected from the cohort of patients eligible for screening at the age of 90 years or above and a retrospective analysis of this group was performed. The outcomes recorded were visual acuity, diabetic retinopathy findings, other ocular findings, and subsequent care in terms of continuing screening or referral for ophthalmology clinical assessment.

For those patients who remained in annual screening and went on to have a second screening episode, data from their most recent screening episode was further analysed to generate outcomes including interval between first and most recent screen episodes, progression of retinopathy, change in quality of fundus image, and also development of new non-DR associated eye conditions detectable on screening. The benefit of screening patients after 90 years of age was then judged based on the occurrence of sight-threatening DR observed at first screening episode aged 90 years as well as on subsequent screening and/or requirement of referral to hospital ophthalmology clinical care and need for laser treatment.

\section{Results}

At the time point of the database search in April 2011, there were 147135 patients in the database of whom 5201 were aged 90 years or over. Conforming to National guideline definitions $46 \%$ of these were eligible for screening. Twohundred patients were randomly selected from this latter group for retrospective study and analysis.

Of the 200 eligible patients, 142 (71\%) were female and $58(29 \%)$ were male. Twenty-one patients were excluded from the analysis-18 did not attend for routine digital screening after attaining the age of 90 years and in 3 an attendance for screening was not possible for medical reasons. The remaining 179 (90\%) patients had attended routine digital screening at least once and the mean number of screens per person was 2 (range 1-6). In terms of first screening episode aged 90 years, this was also the first recorded screen on the digital healthcare database for $57 \%$ of patients. The age of the first recorded screen for the remaining $43 \%$ of patients ranged between 82 and 89 years, with the mean being 87 years of age. In $45 \%$ of patients, the mean duration of DM at time of first screening episode aged 90 years was 10 years (range 1-63 years). The data were unobtainable or unknown for the remaining $55 \%$ of patients.

Figure 1 shows the outcomes of first screening episode aged 90 years for all 179 patients. Mean age was 91 years (range 90-98 years) and the modal Snellen visual acuity (VA) for both eyes was 6/9 (ranges 6/5-NPL). Of the 179 patients, $133(74 \%)$ were placed on annual screening, $38(21 \%)$ were referred routinely or urgently for ophthalmology clinical assessment, and another 8 (5\%) were placed in a digital surveillance clinic.

Of the 133 patients who were placed on annual screening, the final screening outcome was 78 (59\%) had no retinopathy and 55 had either background retinopathy or unassessable image(s).

One key finding of this study is that of the 38 patients referred for ophthalmology clinical assessment, the majority $(n=36 ; 95 \%)$ were not referred for DR (see Figure 2), but for other conditions including AMD, retinal vein occlusion, glaucoma suspect, unassessable images due to cataract or asteroid hyalosis, or due to technical failure owing to insufficient images for grading. Of the five patients referred urgently, four had wet AMD and one had cataract with hand movement (HM) VA. A further key finding is that only two patients had diabetic maculopathy and one received focal laser treatment (aged 91 years).

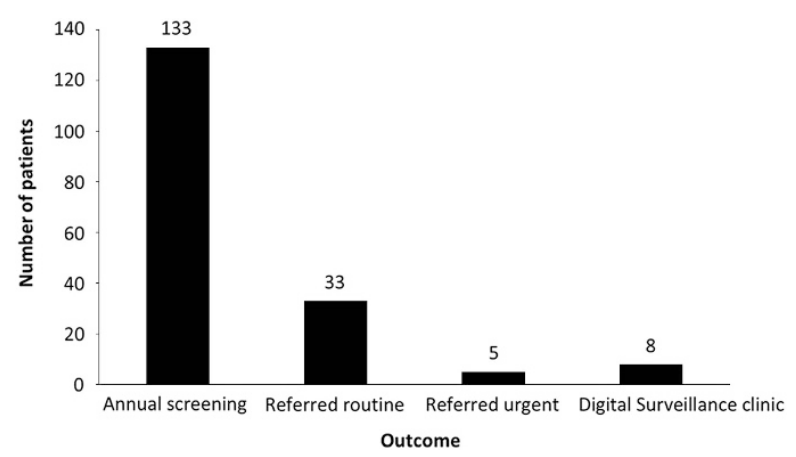

Figure 1 Outcome of first screening episode in persons aged 90 years, $n=179$. Referred routine and referred urgent $=$ referral for ophthalmology clinical assessment.

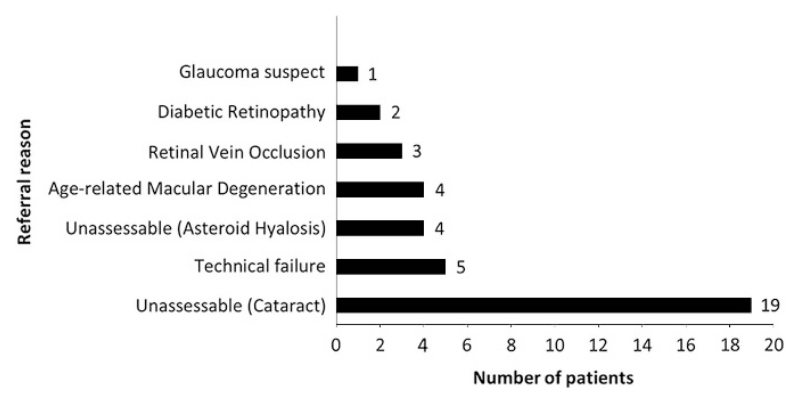

Figure 2 Reasons for referral for ophthalmology clinical assessment, $n=38$. 
'Unassessable images' was the most common reason for referral accounting for 23 out of the 38 (61\%) patients, with cataract accounting for $19(83 \%)$ of these and for $50 \%$ of all referrals for ophthalmology clinical assessment. Of the 23 patients who had unassessable images, 20 were found to have no referable DR upon subsequent ophthalmology clinical assessment. The remaining three patients did not attend their appointment. Of the 19 patients who had unassessable images due to cataract, one eye was unassessable in 12 patients and both eyes were unassessable in the remaining 7 . Five patients subsequently had cataract surgery. The main reasons for patients declining surgery were found to be satisfaction with current vision and risk of complications. One patient died shortly after referral to ophthalmology and their decision on undergoing cataract surgery was not known.

Of the 133 patients placed on annual screening, 93 $(70 \%)$ patients were screened at least once more on annual screening. 40 patients had ceased screening. The reasons for this were: 29 were deceased-(average age of death was 92 years), five had moved out of the area, three were in care ophthalmology and two were permanently inactive. Only one patient remained eligible and did not return in subsequent years. Of the 93 patients who underwent repeat screening (range 1-6 years), the outcomes were: improved in 8 (9\%), stable in $51(57 \%)$, deterioration in 31 (34\%). Of the latter, 19 patients were referred for ophthalmology clinical assessment; none of these for DR. Three patients became unsuitable for digital screening due to mental or physical disability (Figure 3).

\section{Discussion}

In this study, we found that persons who were older than 90 years of age rarely developed sight-threatening DR detected on screening. Regular screening of persons with $\mathrm{DM}$ in this age group may therefore not be justified. The finding is supported by several previous similar studies. For example, a study published in 1997 which evaluated DR in people aged 70 years or older found that 'in spite of the high prevalence of diabetes mellitus in the elderly population, the prevalence of vision threatening diabetic retinopathy, particularly proliferative retinopathy was low. ${ }^{13}$ Furthermore, Klein et $a l^{7}$ reported that in their older-onset group, no participant $>80$ years of age developed proliferative retinopathy. In another study by Stolk et $a l^{8}$ in a total of 6191 patients older than 55 years of age, none were found to have proliferative retinopathy. In another small study it was found that retinopathy rates increased with age, but only until 70 years. ${ }^{14}$

Our findings showed that annual DR screening is very effective in detecting a wide variety of non-DR related eye conditions that are common in the elderly population and the annual screening repeated uptake in the 90 years and

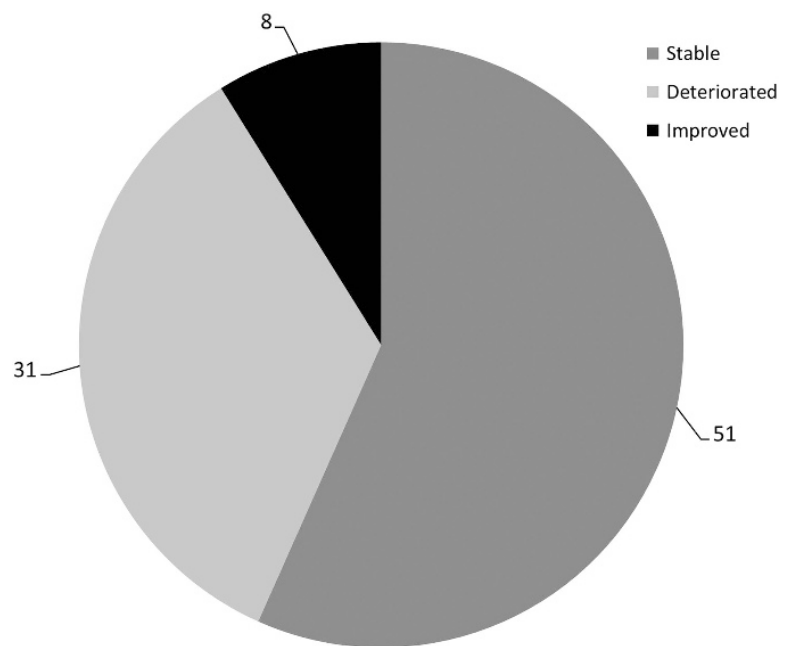

Figure 3 Progression of diabetic retinopathy, change in quality of fundus image and development of new nondiabetic retinopathy-associated eye conditions detected on repeated digital screening, $n=90$. Stable: no change in level of diabetic retinopathy, quality of fundus image, or the presence of nondiabetic retinopathy-associated eye conditions. Deteriorated: deterioration in level of diabetic retinopathy, quality of fundus image, or the presence of new nondiabetic retinopathy-associated eye conditions. Improved: improvement in level of diabetic retinopathy, quality of fundus image, or the resolution of nondiabetic retinopathy-associated eye conditions.

over age group was high at $70 \%$. In total, approximately a fifth of all patients screened were identified as having other eye conditions requiring ophthalmology clinical assessment. In keeping with the published literature, 5,15 the majority (95\%) of patients were referred for ophthalmology clinical assessment after their first screening episode aged 90 years due to non-DR reasons, in particular, media opacity due to cataract which made fundus photography difficult or ungradeable. In our study, cataract(s) interfered with successful digital DR screening in $11 \%$ of first screening episodes aged 90 years and accounted for $50 \%$ of all referrals for ophthalmology clinical assessment. However, despite cataract surgery being readily available and effective in restoring vision, 5 only approximately a quarter of the patients underwent cataract surgery.

This study does not answer the exact age that similar findings would be found as we chose an arbitrary age of $90+$ years. Further studies are required to elucidate whether an age below 90 years could be a suitable cut off. However, digital retinal screening does offer opportunistic identification of important non-DR related conditions and therefore is an effective way of preventing and restoring visual loss and maintaining the best possible quality of life in this population group. ${ }^{16}$ Although valuable, this, however, is not the intended purpose of DR screening. The current mission statement of the NHS Diabetic Eye Screening Programme is to 
reduce the risk of sight loss among people with diabetes by the early detection and treatment, if needed, of sightthreatening DR. We found a low detection rate of maculopathy in our screened population of 90 years at $1 \%$ and this is a substantially lower rate than $5 \%$ of screens for maculopathy in systematically screened diabetic populations starting at the age of 12 years.

Considering the above, our data raise the question as to whether systematic screening should stop at the age of 90 years. It may be argued that it is more appropriate and cost effective for patients over 90 years of age to be encouraged to attend their optometrist on a regular basis rather than with the DR screening programme. This would still enable the detection of maculopathy but would also be better suited for the detection of much more prevalent non-DR related eye conditions in this group of patients. The optometrist, who could conduct a full ophthalmic examination, would also be better placed to refer these elderly patients directly to specific eye clinics, for example, cataract, glaucoma, or macular clinics, as and when appropriate.

In summary, a cut-off age of 90 years was arbitrarily selected in this instance and therefore lower age bands under 90 years have not been considered as part of this study. Similar analyses could be performed in future studies using larger sample sizes to gauge the utility of systematic DR screening in other age brackets. In terms of persons over 90 years of age, our data and the evidence in the published literature suggest a low utility of the screening programme at detecting sight-threatening DR. Altogether with the high prevalence of non-DR related eye conditions, we feel that the intended purpose of the NHS Diabetic Eye Screening Programme is not fulfilled in this age group of patients and screening may be better performed in optometric practice.

\section{Summary}

What was known before

- There have been several previous small studies that have looked at diabetic eye screening in persons over 70 years of age but none that have looked specifically at screening in a population over 90 years of age to ascertain the value of systematic screening in this age group of patients. UK screening programmes for other conditions (eg, breast and cervical screening) have upper age limits but this has not been considered previously for diabetic eye screening.

\section{What this study adds}

- Screening in a population group aged 90 years and over identifies a significant proportion of patients with visual threatening eye conditions, not diabetic retinopathy related which can be treated. The important observation for screening is that sight-threatening diabetic retinopathy is rare in this age group of patients. In view of the current mission statement of the NHS Diabetic Eye Screening Programme to prevent visual loss from diabetic retinopathy, we suggest that systematic diabetic retinopathy screening could stop at the age of 90 years and screening of this population could become opportunistic, for example, within optometric practice.

\section{Conflict of interest}

The authors declare no conflict of interest.

\section{References}

1 http://www.diabetes.org.uk/Documents/About\%20Us/ annual-reports / diabetes-uk-report-financialstatements-2012-signed-0613.pdf. Accessed on 7 November 2013.

2 Wild S, Roglic G, Green A, Sicree R, King H. Global prevalence of diabetes: estimates for the year 2000 and projections for 2030. Diabetes Care 2004; 27(5): 1047-1053.

3 Green A, Christian Hirsch N, Pramming SK. The changing world demography of type 2 diabetes. Diabetes Metab Res Rev 2003; 19(1): 3-7.

4 Benbow SJ, Walsh A, Gill GV. Diabetes in institutionalised elderly people: a forgotten population? BMJ 1997; 314: 1868-1869.

5 Quillen DA. Common Causes of Vision Loss in Elderly Patients. Am Fam Physician 1999; 60: 99-108.

6 Fong DS, Aiello L, Gardner TW, King GL, Blankenship G, Cavallerano JD et al. Diabetic Retinopathy. Diabetes Care 2003; 26: s99-s102.

7 Klein R, Klein BEK, Moss SE. Epidemiology of proliferative diabetic retinopathy. Diabetes Care 1992; 15: 1875-1891.

8 Stolk RP, Vingerling JR, de Jong PTVM, Dielemans I, Hofman A, Lamberts SW et al. Retinopathy, glucose, and insulin in an elderly population. The Rotterdam Study. Diabetes 1995; 44: 11-15.

9 Sommer A, Tielsch JM, Katz J, Quigley HA, Gottsch JD, Javitt JC et al. Racial differences in the cause-specific prevalence of blindness in East Baltimore. N Engl J Med 1991; 325: 1412-1417.

10 Cahill M, Halley A, Codd M, O'Meara N, Firth R, Mooney D et al. Prevalence of diabetic retinopathy in patients with diabetes mellitus diagnosed after the age of 70 years. Br J Ophthalmol 1997; 81: 218-222.

11 Li X, Wang Z. Prevalence and incidence of retinopathy in elderly diabetic patients receiving early diagnosis and treatment. Exp Ther Med 2013; 5: 1393-1396.

12 Valsania P, Warram JH, Rand LI, Krolewski AS. Different determinants of neovascularization on the optic disc and on the retina in patients with severe nonproliferative diabetic retinopathy. Arch Ophthalmol 1993; 111: 202-206.

13 Hirvelä H, Laatikainen L. Diabetic retinopathy in people aged 70 years or older. The Oulu Eye Study. Br J Ophthalmol 1997; 81: 214-217.

14 Khandekar R, Al Lawatii J, Mohammed AJ, Al Raisi A. Diabetic retinopathy in Oman:a hospital based study. Br J Ophthalmol 2003; 87: 1061-1064.

15 Klaver CC, Wolfs RC, Vingerling JR, Hofman A, de Jong PT et al. Age-specific prevalence and causes of blindness and visual impairment in an older population. The Rotterdam Study. Arch Ophthalmol 1998; 116: $653-658$.

16 Sinclair AJ, Turnball CJ, Croxson SCM. Document of care for older people with diabetes. Postgrad Med F 1996; 72: 334-338. 